Check for updates

Cite this: Phys. Chem. Chem. Phys., 2021, 23, 6641

Received 23rd November 2020 Accepted 11th February 2021

DOI: 10.1039/d0cp06068b

rsc.li/pccp

\title{
Molecular features toward high photo-CIDNP hyperpolariztion explored through the oxidocyclization of tryptophan $\dagger$
}

\author{
Felix Torres, (D) ${ }^{a}$ Alexander Sobol, $\stackrel{+}{a}^{\mathrm{a}}$ Jason Greenwald, ${ }^{\mathrm{a}}$ Alois Renn, ${ }^{a}$ \\ Olga Morozova, $\mathbb{D}^{\mathrm{b}}$ Alexandra Yurkovskaya $\mathbb{D}^{\mathrm{b}}$ and Roland Riek*a
}

\begin{abstract}
Photo-chemically induced dynamic nuclear polarization (photo-CIDNP) is a promising solution to the inherent lack of sensitivity in NMR spectroscopy. It is particularly interesting in biological systems since it operates in water, at room temperature, and it can be repeated if the bleaching of the system can be controlled. However, the photo-CIDNP signal enhancement is well below those of other hyperpolarization techniques. While DNP, PHIP, and SABRE reach polarization enhancements of $10^{3}$ to $10^{4}$-fold, photo-CIDNP enhancement is typically only one order of magnitude for ${ }^{1} \mathrm{H}$ and two orders of magnitude for ${ }^{13} \mathrm{C}$ in the amino-acids tryptophan and tyrosine. Here we report on a photo-oxidation product of tryptophan that is strongly photo-CIDNP active under continuous wave light irradiation. In conjunction with the dye Atto Thio 12, a ${ }^{1} \mathrm{H}$ signal enhancement of 120 -fold was observed on a $600 \mathrm{MHz}$ spectrometer, while at $200 \mathrm{MHz}$ the enhancement was 380 -fold. These enhancements in signal to noise correspond to a reduction in measurement time of 14400-fold and 144400-fold, respectively. The enhancement for ${ }^{13} \mathrm{C}$ is estimated to be over 1200 -fold at $600 \mathrm{MHz}$ which corresponds to an impressive measurement time reduction of 1440000 -fold. This photo-CIDNP active oxidation product of tryptophan has been identified to be $3 \alpha$-hydroxypyrroloindole. The reasons for its improved signal enhancement compared to tryptophan have been further investigated.
\end{abstract}

\section{Introduction}

Nuclear magnetic resonance (NMR) is extensively used in biology to obtain structural information at atomic resolution. However, despite impressive technological innovations including the increasing strength of magnetic fields, the introduction of the cryo-probe and improvements in the pulse-sequences, the issue of sensitivity remains the major barrier to applying NMR to a broader set of problems in biology. NMR is inherently insensitive because of the low Boltzmann population difference between up and down nuclear spins at room temperature. To overcome this hurdle, several hyperpolarization techniques have been proposed during the last 60 years such as dynamic nuclear polarization (DNP) ${ }^{1,2}$ para-hydrogen induced polarization (PHIP), ${ }^{3}$ signal

\footnotetext{
${ }^{a}$ Laboratory of Physical Chemistry, Swiss Federal Institute of Technology, ETH-Hönggerberg, $\mathrm{CH}-8093$ Zürich, Switzerland.

E-mail: roland.riek@phys.chem.ethz.ch

${ }^{b}$ International Tomography Center, Siberian Branch of the Russian Academy of Science, Novosibirsk 630090, Russia and Novosibirsk State University,

Novosibirsk 630090, Russia

$\dagger$ Electronic supplementary information (ESI) available. See DOI: 10.1039/ d0cp06068b

\# Deceased.
}

amplification by reversible exchange (SABRE), ${ }^{4,5}$ and chemically induced dynamic nuclear polarization (CIDNP), the last of which was independently discovered by Bargon et al. ${ }^{6}$ and Ward et al. ${ }^{7}$ in 1967. The photo-CIDNP setup is straightforward since it only requires irradiation of the sample, which is here performed thanks to the combination of a light source and an optic guide. The CIDNP theory is well established and relies on the radical pair mechanism proposed by Closs Kaptein and Oosterhoff in 1969.8,9 The extension by Adrian, ${ }^{10}$ which uses the Noyes ${ }^{11}$ diffusion theory constitutes an approximate theoretical framework including the present study.

This study was put in motion by a serendipitous observation, while measuring the photo-CIDNP activity of fluorescein ${ }^{12}$ and Atto Thio 12 (AT12) $^{13}$ on the amino acid tryptophan (Fig. 1, see also below) under continuous wave (CW) irradiation. After a series of photo-CIDNP measurements on the same tryptophan/ AT12 sample, a number of new peaks appeared in the light irradiated spectra that remained undetectable in the dark spectra, evidence of an unexpected polarization (Fig. 1). Interestingly, traces of this photo-CIDNP-active product can also be found in the literature, notably in Okuno et al., ${ }^{14}$ where a photo-CIDNP active impurity is visible for the spectrum of irradiated tryptophan in the presence of fluorescein and 


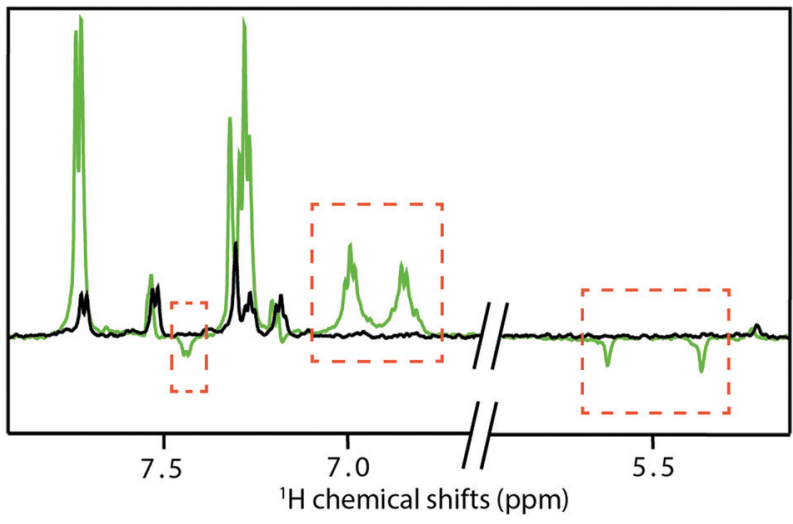

Fig. 1 Unexpected polarisation of a trace degradation product. PhotoCIDNP of a 1 day old $1 \mathrm{mM}$ tryptophan sample measured by ${ }^{1} \mathrm{H}$ 1D NMR at 14.1 $\mathrm{T}$ (i.e. $600 \mathrm{MHz}{ }^{1} \mathrm{H}$ NMR frequency), irradiated in the presence of $25 \mu$ M AT12 with a 1 W $532 \mathrm{~nm}$ laser. The dark spectrum is in black and the irradiated spectrum is in green. The red dashed boxes emphasize the highly polarized signals from an unknown molecule.

3-iodo-1-propanol. The highly polarized impurity was obviously suspected to be a photoinduced degradation product of tryptophan. Indeed, we identified it as an oxidocyclization product of tryptophan that results from the generation of ${ }^{1} \mathrm{O}_{2}$ by the triplet state of AT12. Subsequently, the resulting $3 \alpha$-hydroxypyrroloindole was successfully synthesized, characterized and its photo-CIDNP activity measured. Moreover, the combination of CW photo-CIDNP, time-resolved (TR) photo-CIDNP experiments and density functional theory (DFT) simulations provided valuable information about the reasons for such an improvement of the signal enhancement in the $3 \alpha$-hydroxypyrroloindole as compared to that of tryptophan. The relevant parameters for CIDNP such as the $\Delta g$ and the hyperfine coupling constants (HFCCs) were related to the observed anomalous lines in TR-photo-CIDNP experiments. Furthermore, insights into the reaction kinetics of the photo-CIDNP reaction emphasized the possible importance of conformation, and the molecular geometry for photo-CIDNP activity. All these mechanistic studies were done in order to evaluate the potential of using CW photoCIDNP as a signal enhancement tool in biologically and chemically focused NMR.

\section{Theoretical background for photo-CIDNP}

The anomalous lines observed in the CIDNP experiment are explained by the radical pair mechanism. ${ }^{9}$ The following section provides a brief overview about the photo-CIDNP theory. Nevertheless, several good and recent reviews should be consulted for a more extensive description of this phenomenon, such as Okuno et al., and Morozova et al. ${ }^{15,16}$

The radical pair can be generated by a light-excited dye that undergoes intersystem crossing into a triplet state. ${ }^{17}$ As a consequence of its electronic structure, the triplet state dye is long-lived and highly redox-active. Hence, when the ${ }^{\mathrm{T}} \mathrm{D}$ collides with an aromatic molecule, it can generate a radical pair (Fig. 2, step a). The newly formed radical pair is in a triplet state, where the Pauli principle applies and forbids the electron back transfer. The recombination of the radical pair is only possible when the pair has evolved into a singlet state. When the two radicals diffuse away from each other, the electron-exchange coupling vanishes (Fig. 2, step b) and the triplet-singlet mixing happens, according to eqn (1):

$$
\omega_{\mathrm{ISC}}=\left(g_{\mathrm{M}}-g_{\mathrm{D}}\right) \mu_{\mathrm{B}} B_{0}+\sum A_{\mathrm{M}, \mathrm{a}} m_{\mathrm{M}, \mathrm{a}}-\sum A_{\mathrm{D}, \mathrm{b}} m_{\mathrm{D}, \mathrm{b}}
$$

with $g_{\mathrm{M}, \mathrm{D}}$ being the $g$-factors of the two radicals, $\mu_{\mathrm{B}}$ the Bohr magneton, and $A$ the HFCC, which are summed over the nuclei $\mathrm{a}$ and $\mathrm{b}$ of the molecules $\mathrm{M}$ and the dye $\mathrm{D}$, respectively. The equation yields two different frequencies for the different nuclei spin states $( \pm 1 / 2)$ denoted as $m$. Therefore, if the radical pair is reformed after a time $\tau$, the probability that it is in a singlet state is given by eqn (2):

$$
|\langle S \mid \psi(\tau)\rangle|^{2}=\sin ^{2}\left(\omega_{\text {ISC }} \tau\right)
$$

The two spin correlated entities forming the radical pairs have an increased probability of re-encounter after separation, ${ }^{11}$ which follows a dependency of the order or $t^{-3 / 2}$. The singlet radical pairs, recombine to generate ground state dye (D) and molecule (M) (Fig. 2, step c). The resulting geminate polarization $\left(P^{\mathrm{G}}\right)$ is the difference in $\alpha$ and $\beta$ spin populations originating from the probabilities of recombining for a spin $\alpha$ and $\beta$, respectively (eqn (3)). ${ }^{18}$

$$
P^{\mathrm{G}} \sim\left|\omega_{\mathrm{ISC}, \alpha}\right|^{1 / 2}-\left|\omega_{\mathrm{ISC}, \beta}\right|^{1 / 2}
$$

where $\left|\omega_{\text {ISC }, \alpha}\right|^{1 / 2}$ and $\left|\omega_{\text {ISC }, \beta}\right|^{1 / 2}$ are the populations of $\alpha$ and $\beta$ spins obtained by integration of eqn (2) over the timedependent recollisional probability.

The radical pairs that recollide in a $\mathrm{T}$ spin state cannot recombine and therefore diffuse apart in the bulk solution (Fig. 2, step d). These free radicals can collide with a new partner and form a radical pair (F-pair), in singlet or triplet states. The singlet state pairs recombine directly and have no effect on the final polarization. The $\mathrm{T}$ radical pair can generate polarization again by the nuclear spin-dependent triplet-singlet electron spin mixing. The polarization arising from the repeated cycles of free $\mathrm{M}$ and $\mathrm{D}$ radical collisions is called F-pair polarization ${ }^{19-21}\left(P^{\mathrm{F}}\right)$, and is added to the $P^{\mathrm{G}}$ to yield the final polarization $(P)$.

In order to reach the maximal possible polarization, the study was conducted under continuous wave laser irradiation on the order of seconds. Hence, the hyperpolarization present after $\mathrm{CW}$ irradiation in the second timescale is a mix resulting from the $P^{\mathrm{G}}$ and the $P^{\mathrm{F}}$. However, only the use of time-resolved (tr) photo-CIDNP enables us to observe the pure geminate polarization $^{16,22,23}$ necessary to obtain the fine information about the key physical properties governing the singlet-triplet mixing of the radicals such as the $g$-factors and the HFCCs. As described in this section the intensity of photo-CIDNP spectra is driven by the efficiency of the quenching of the triplet state dye by the molecule of interest and the magnetic parameters such as $g$-factor and the HFCCs. 


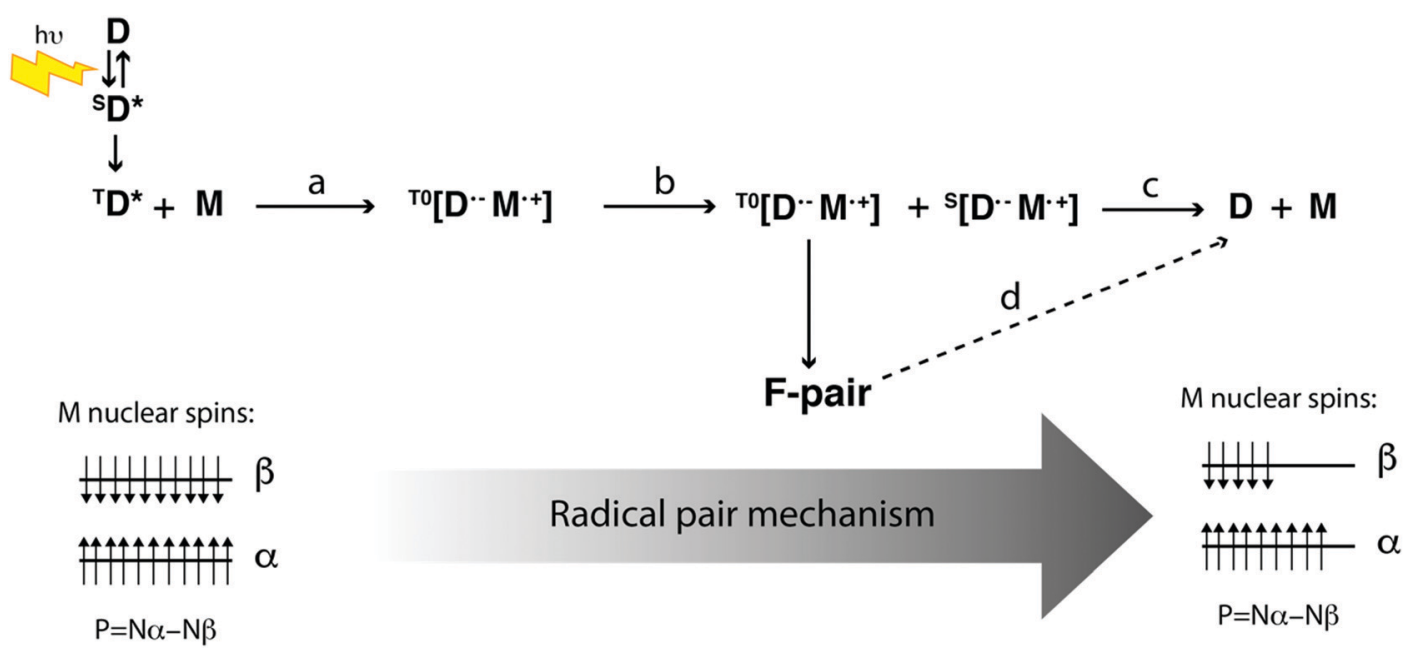

Fig. 2 Schematic representation of the photo-CIDNP mechanism. The different steps are described in more detail in the text. D is the symbol of the photosensitizer and $\mathrm{M}$ isthe symbol for any aromatic molecule that can form a radical pair with $\mathrm{D}$. The nuclear spins before and after radical pair mechanism are represented to emphasize the polarization $(P)$, which is the difference between the nuclear spin populations, $N \alpha$ and $N \beta$.

\section{Materials and methods}

The NMR measurements were performed at $298 \mathrm{~K}$ either on a Bruker Avance III $600 \mathrm{MHz}$ spectrometer equipped with a cryoprobe or on a Bruker Avance $200 \mathrm{MHz}$ spectrometer equipped with a room temperature probe. The irradiation of AT12 samples was performed with a Coherent Verdi V10 diodepumped solid state laser emitting at a wavelength of $532 \mathrm{~nm}$. The laser used for the fluorescein samples was a Thorlabs L450P1600MM, with a diode laser emitting at $450 \mathrm{~nm}$. The laser light was coupled (using appropriate coupling optics) to an optical fiber (Thorlabs, FG950UEC) of length $10 \mathrm{~m}$ and a diameter of $0.95 \mathrm{~mm}$. The end of the fiber was inserted into the sample solution in a $3 \mathrm{~mm}$ NMR tube to a depth of about $5 \mathrm{~mm}$ above the NMR coil region. The TR-photo-CIDNP experiments were performed on a Bruker DPX $200 \mathrm{MHz}$ NMR spectrometer, and irradiation was performed with a COMPEX Lambda Physik $\mathrm{XeCl}$ excimer laser (wavelength $308 \mathrm{~nm}$, and pulse energy up to $150 \mathrm{~mJ})$.

The HPLC analysis and initial purification was performed on an Agilent Technologies ${ }^{\circledR} 1200$ LC system using a semipreparative Kinetex ${ }^{\circledR}$ C18 column. The runs were performed with an acetonitrile $(0.1 \%$ trifluoroacetic acid, TFA) gradient going from 3 to $30 \%$ in water (1\% TFA) in $15 \mathrm{~min}$ at a flow rate of $3 \mathrm{~mL} \mathrm{~min}^{-1}$, at $40{ }^{\circ} \mathrm{C}$. The UV spectra were recorded with a Jasco ${ }^{\circledR}$ V-650 UV-vis spectrometer at a concentration of $0.01 \mathrm{mM}$. Tyrosine and tryptophan (Sigma) were prepared as stock solutions of $0.2 \mathrm{mg} \mathrm{ml}^{-1}$ and $0.18 \mathrm{mg} \mathrm{ml}^{-1}$, respectively, in a $0.1 \mathrm{M}$ sodium/potassium phosphate buffer ( $\mathrm{pH}$ 7.1) with $5 \%$ $\mathrm{D}_{2} \mathrm{O}$. The stock solution of Atto Thio 12 (AT12) was $1 \mathrm{mg} \mathrm{ml}^{-1}$ in $\mathrm{H}_{2} \mathrm{O}$. To prevent dye quenching the enzyme cocktail of glucose oxidase (Go, $120 \mathrm{kDa}$ ), catalase (Cat, $240 \mathrm{kDa}$ ) and D-glucose (G, $180 \mathrm{Da})$ was used at a concentration of $14 \mathrm{nM}$ for each enzyme and $2.5 \mathrm{mM}$ of glucose, as described elsewhere. ${ }^{12,24}$ The stock solutions were $0.25 \mu \mathrm{M}$ for Go and $0.16 \mu \mathrm{M}$ for Cat, respectively. The glucose stock solution was $500 \mathrm{mM}$ in $\mathrm{D}_{2} \mathrm{O}$ with $0.02 \% \mathrm{NaN}_{3}$. All the samples were prepared in $100 \mathrm{mM}$ of a $\mathrm{KPO}_{4}$ buffer at $\mathrm{pH}=7.1$ with either $20 \mu \mathrm{M}$ of AT12 or $25 \mu \mathrm{M}$ of fluorescein, and $100 \mu \mathrm{M}$ of target molecule (i.e. tyrosine, tryptophan or HOPI), unless otherwise specified. The sample for TRphoto-CIDNP was prepared using $4 \mathrm{mM}$ of $\mathrm{HOPI}$ and $4 \mathrm{mM}$ of TCBP dye at $\mathrm{pH}=7.5$.

\section{Results and discussion}

\section{HOPI characterization}

In order to identify the molecule that showed the large polarization enhancement depicted in Fig. 1, the irradiated samples in which the highly polarized impurities could be observed were pooled together and purified by reversed-phase HPLC. Several peaks from the chromatogram were collected separately and screened by NMR with the photo-CIDNP experiment. More than one HPLC peak had significant photo-CIDNP signal enhancement and after identifying the peak with the highest photo-CIDNP signal enhancement, state-of-the-art NMR experiments were used to characterize its chemical structure (Fig. S1-S3, ESI $\dagger$ ). In addition, the product was characterized by ESI-TOF mass spectrometry yielding a positive $\mathrm{m} / \mathrm{z}$ of 221 . Dyes can relax from an excited singlet state towards a triplet state towards intersystem crossing. This later state relaxes very slowly to the ground state and is therefore prone to react with oxygen to form the very oxidative singlet oxygen (Fig. 3). As the literature provided many characterized tryptophan oxidation products as potential candidates for the unknown molecules, ${ }^{25-27}$ these species were investigated further. The $\mathrm{m} / \mathrm{z}$ ratio suggests the addition of an oxygen atom, and the $1 \mathrm{D}{ }^{1} \mathrm{H}-\mathrm{NMR}$ spectrum shows that all the hydrogens on the benzo group remain, eliminating all the hydroxy-tryptophans as candidates. The $3 \alpha-$ hydroxypyrroloindole (HOPI) depicted in Fig. 3 is consistent with the NMR data and the $\mathrm{m} / \mathrm{z}$ ratio from MS. The reaction mechanism leading to HOPI involves an epoxy intermediate that can form on both sides of the double bond of the pyrrole moiety of tryptophan. The direct consequence of this is the presence of 

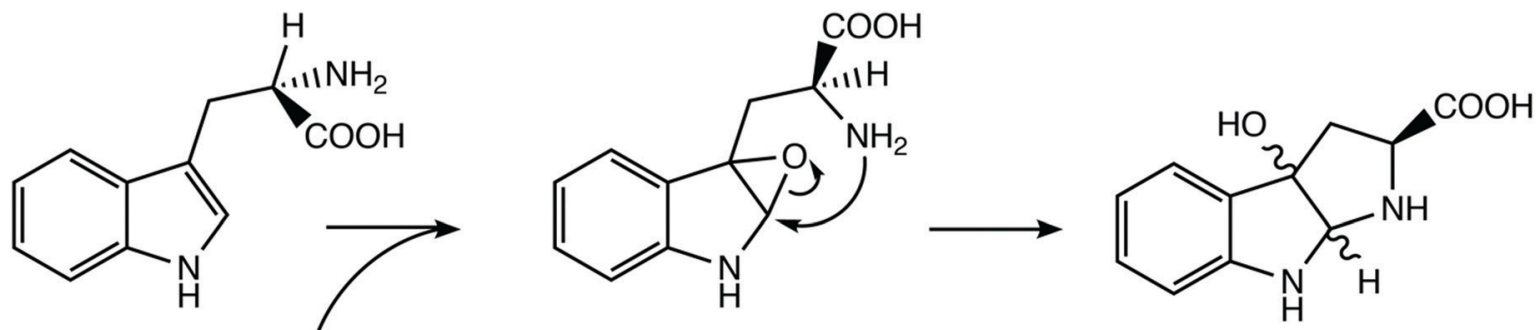

two diastereoisomers when the nitrogen of the amine group opens the epoxide following an SN2 mechanism. This last step leads to cyclization. The two diastereoisomers have been previously characterized. ${ }^{28}$ The trans isomer is more polar than the cis, based on the C18 HPLC retention times (Fig. S4, ESI $\dagger$ ). Moreover, the chemical shifts associated with the different diastereoisomers were consistent with the ones previously described in the literature ${ }^{28}$ in particular for the $\mathrm{H}_{\beta}$ and the $\mathrm{H}_{\alpha}$. Next, the HOPI diastereoisomer pair was synthesized according to the formerly described procedure. ${ }^{29}$ The full experimental protocol for the HOPI synthesis is detailed in the dedicated ESI. $\dagger$ The synthesized products exhibited the same NMR spectra and polarization properties during photo-CIDNP experiments as observed for the degradation products of tryptophan (compare Fig. 1 with Fig. 4 A and B).

\section{Photo-CIDNP findings for HOPI}

The oxidocyclization of tryptophan into HOPI greatly affects its CW-photo-CIDNP performance. Of particular interest is the strong AT12-based polarization found for ${ }^{1} \mathrm{H}$ with a factor of 73 for trans-HOPI and 68 for cis-HOPI at $100 \mu \mathrm{M}$ HOPI concentration in a $14.1 \mathrm{~T}$ magnetic field (i.e. $600 \mathrm{MHz}$ ${ }^{1} \mathrm{H}$ NMR frequency). Similarly, polarization in the presence of fluorescein yields a factor of -70 for trans-HOPI and -47 for cis-HOPI (Fig. 4A, B and Table 1). Interestingly, both HOPIs show a strong concentration-dependence of the signal enhancement but display contrary behaviours for the two diastereoisomers. At the lower concentration of $20 \mu \mathrm{M}$, transHOPI shows an enhancement of 120 for the $\mathrm{H}_{5}$ resonance, whereas for cis-HOPI it is only ca. 40 (Fig. 4C), while at a concentration of $c a .0 .5 \mathrm{mM}$ both show a similar enhancement of $c a$. 60 . At $4.7 \mathrm{~T}(200 \mathrm{MHz})$ the trans-HOPI is enhanced by 380 -fold in the presence of AT12, and 160-fold in the presence of fluorescein (Table 1). The signal enhancement for the transHOPI at $4.7 \mathrm{~T}$ is illustrated in Fig. 4C. In contrast to the singlescan photo-CIDNP $1 \mathrm{D}{ }^{1} \mathrm{H}$ NMR experiment the reference dark spectrum with a factor of about three-fold less signal to noise ratio took $16 \mathrm{~h}$ to acquire with 11520 scans, a time difference of five orders of magnitude.

While tryptophan is typically better polarized with fluorescein than AT12, the trans-HOPI and to a lesser extend the cis-HOPI are equally hyperpolarized after irradiation in the presence of AT12 or fluorescein dyes (Table 1). Furthermore, the polarization sign for HOPI is inverted with fluorescein compared to that of AT12 (Fig. 4A and B). Moreover, the pattern of the signal enhancement is perturbed. The photo-CIDNP spectrum of tryptophan with AT12 shows only absorptive lines for the aromatic protons and emissive lines for the $\mathrm{H}_{\beta}$ (Fig. S5, ESI $\dagger$ ), while the HOPI spectrum presents both absorptive and emissive lines for the aromatics and is emissive for the newly aliphatic $\mathrm{H}_{2}$ (Fig. 4A and B).

The finding that dyes react preferentially with some molecules over others, yielding to preferential polarization, has already been reported. This is in particular the case for fluorescein and tryptophan, which are a better pair than fluorescein and tyrosine $^{12}$ and conversely for tyrosine, which is more efficiently hyperpolarized with AT12 than tryptophan. ${ }^{13}$ In the high-field limit for fast tumbling molecules this difference is attributed to the offset in the Zeeman splitting $(\Delta g)$ of the two single electrons of the respective dye and molecule (eqn (1)), while the changes in sign and relative intensities are explained by the changes in the HFCC values upon chemical structure modification.

\section{Photo-CIDNP of ${ }^{13} \mathrm{C}$ resonances of HOPI}

It is important to note that the here-documented polarization enhancements are for ${ }^{1} \mathrm{H}$, while it is common that the polarization enhancements are reported for ${ }^{13} \mathrm{C}$ or other nuclei, which have either a lower gyromagnetic ratio or larger HFCC when compared with ${ }^{1} \mathrm{H}$. The anisotropy of the ${ }^{13} \mathrm{C}$ HFCC needs to be considered for a proper analysis since their anisotropic character is much more pronounced than for hydrogen. For the carbons C5, C7, and C2 we could calculate HFCC values for the $z$-axis (in the molecular frame) of 25.1, 22.3, and 27.9 Gauss, respectively. Thus, for the following 3 reasons it is expected that HOPI will display an even stronger enhancement for ${ }^{13} \mathrm{C}$ : (1) its gyromagnetic ratio is 
A)

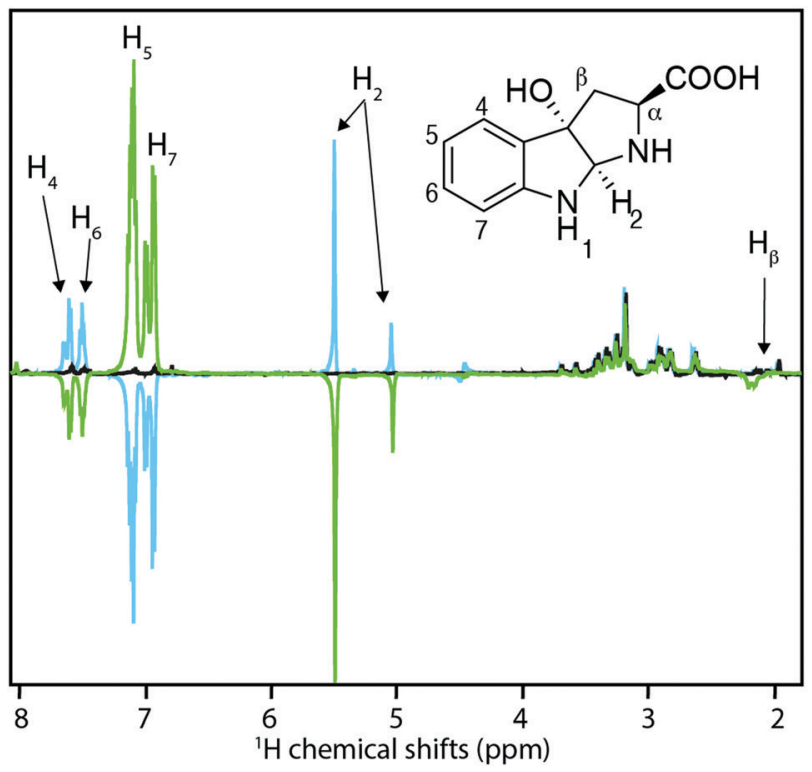

C)

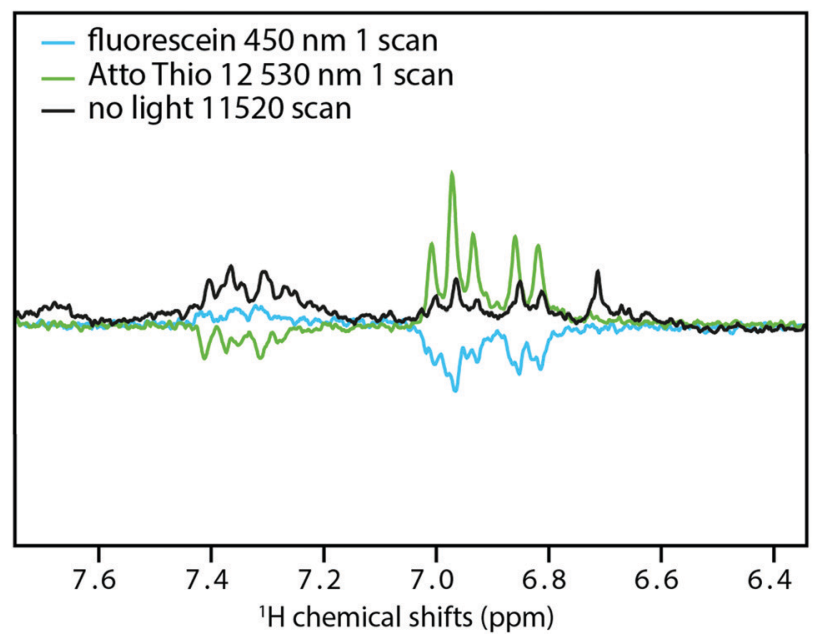

B)

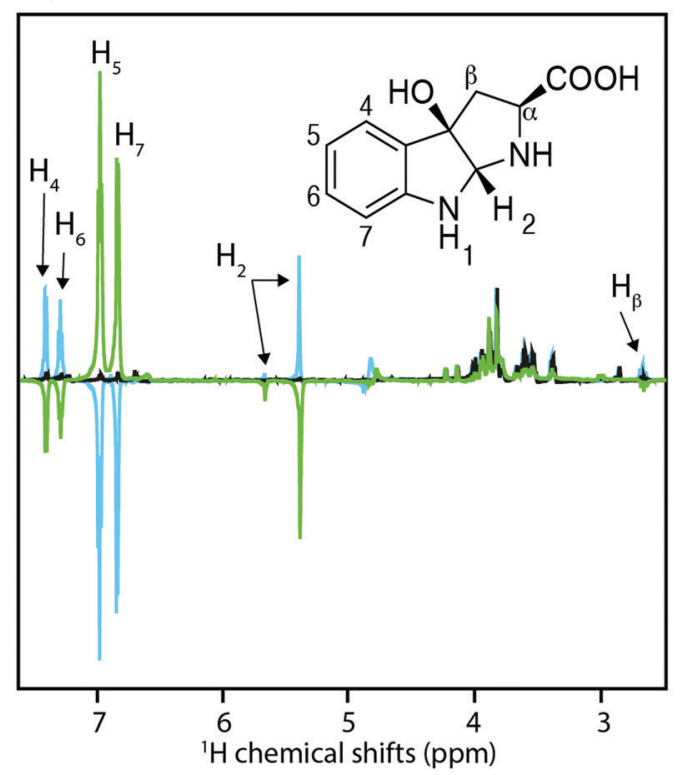

D)

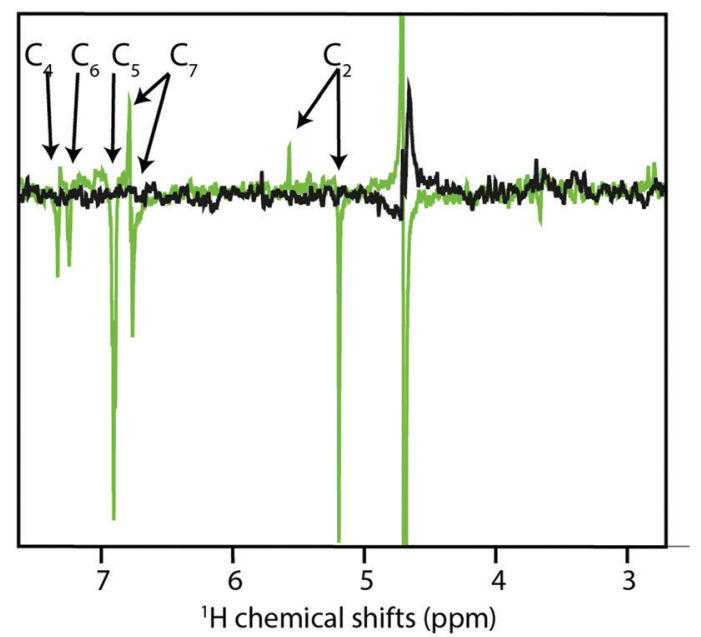

Fig. 4 Photo-CIDNP ${ }^{1} \mathrm{H}$ 1D NMR spectra of HOPI, $100 \mu \mathrm{M}$, in the presence of AT12, $20 \mu \mathrm{M}$, (green lines) or fluorescein, $25 \mu \mathrm{M}$ (blue lines). In black are the dark ${ }^{1} \mathrm{H}$ 1D NMR spectra. The irradiation was performed for $4 \mathrm{~s}$ at $1 \mathrm{~W}$ power level, and wavelengths of $450 \mathrm{~nm}$ for fluorescein and $532 \mathrm{~nm}$ for AT12. (A) trans-HOPI at $600 \mathrm{MHz}$ and (B) cis-HOPI at $600 \mathrm{MHz}$. Note that trans-HOPI displays two $\mathrm{H}_{2}$ and $\mathrm{H}_{7}$ signals due to different conformations. (C) trans$\mathrm{HOPI}$ at $200 \mathrm{MHz}$. The dark spectrum was accumulated over 11520 scans. For representation purposes the spectra were normalized to the noise level, hence the dark spectra are scaled by a factor of 0.0093 , which correspond to $1 / \sqrt{ } 11520$. (D) Photo-CIDNP of ${ }^{13} \mathrm{C}$ detected on the attached ${ }^{1} \mathrm{H}$ of naturally abundant trans-HOPI at $600 \mathrm{MHz}$. The dark spectrum (no light) was accumulated over 2560 scans. For representation purposes the spectra were normalized to the noise level; hence, the dark spectrum is scaled by 0.02 , which corresponds to $1 / \sqrt{ } 2560$. The (very weak) signal at 3.8 ppm of the dark spectrum is due to the sugars, which are at a concentration of $2.5 \mathrm{mM}(\mathrm{ESI} \dagger)$, and were used for the cross-calculation of the signal enhancement.

3.98-fold lower than that of ${ }^{1} \mathrm{H},(2)$ the ${ }^{13} \mathrm{C}$ HFCCs are at least a factor of 2 higher than that for the protons (Tables $\mathrm{S} 2$ and S3, $\mathrm{ESI} \dagger$ ) the longitudinal relaxation of ${ }^{13} \mathrm{C}$ is slower than that of ${ }^{1} \mathrm{H}$ yielding less signal decay during the $\mathrm{CW}$ irradiation. Hence, at least a factor of 8 more is expected. Indeed, a ${ }^{13} \mathrm{C}$ signal enhancement of 1217fold is estimated in a 1D single scan photo-CIDNP experiment on trans-HOPI and AT12 performed on a $600 \mathrm{MHz}$ spectrometer starting with ${ }^{13} \mathrm{C}$ (steady state) magnetization that is detected on its covalently bounded ${ }^{1} \mathrm{H}$ via a refocusing $\mathrm{INEPT}^{31}$ (Fig. S14B ESI $\dagger$ and Fig. 4D). In this case, only an estimation was possible since the trans-HOPI sample contained naturally abundant ${ }^{13} \mathrm{C}$, yielding a dark reference spectrum of extremely low signal intensity. It is noted that the estimated signal enhancement equals a $10^{6}$-fold reduction in measurement time. The irradiated spectrum measured in 1 scan (5 seconds) would have required 1440000 scans for the dark spectrum, which corresponds to 2000 hours of measurement or approximately 83 days.

\section{$g$-Factor differences $(\Delta g)$}

In the following, the Zeeman splitting, the HFCC values and reaction kinetics of both HOPI diastereoisomers are investigated in some detail starting with $\Delta g$. As shown above in the 
Table 1 Photo-CIDNP ${ }^{1} \mathrm{H}$ signal enhancement with different dyes and magnetic fields

\begin{tabular}{lllll}
\hline & $\begin{array}{l}\text { Fluorescein } \\
\text { AT12 at 14.1 T } 14.1 \mathrm{~T} \\
(600 \mathrm{MHz})\end{array}$ & $\begin{array}{l}\text { AT12 at } \\
(600 \mathrm{MHz})\end{array}$ & $\begin{array}{l}\text { Fluorescein } \\
(200 \mathrm{MHz})\end{array}$ & $\begin{array}{l}\text { at } 4.7 \mathrm{~T} \\
(200 \mathrm{MHz})\end{array}$ \\
\hline Molecule & & -70 & 380 & -160 \\
trans-HOPI & 73 & -42 & N.D. & N.D. \\
cis-HOPI & 68 & 53 & 90 & 225 \\
TRP & 18 & &
\end{tabular}

The signal-to-noise enhancement factors correspond to the strongest aromatic resonance of HOPI (H5) and tryptophan (H4), both at $100 \mu \mathrm{M}$.

photo-CIDNP theory section, in the high-field limit for fast tumbling molecules (i.e. $<1 \mathrm{~ns}$ ), the singlet-triplet state mixing is mainly driven by the $\Delta g$. This parameter results from the spinorbital coupling of the unpaired electron with the SOMO. The oxidocyclization of the HOPI transforms the aromatic system from an indole ring to an aniline-like aromatic ring after the conversion of the pyrrole part of the indole group into a pyrrolidine. This modification of the shape and expansion of the SOMO is suggested to be the reason for the different $g$-factor values of tryptophan and HOPI. An experimental support of the $g$-factor modification through the chemical reaction is the sign alteration of the HOPI photo-CIDNP when the dye is changed from fluorescein to AT12 (Fig. 3A and B). According to the Kaptein rules, ${ }^{32}$ this polarization sign alteration is explained by the dye-dependent sign alteration of the radical pair $\Delta g$. Indeed, no other conditions are changed between the two experiments except the dye. Furthermore, the observed magnetic fielddependence of the enhancements support the important role of the $\Delta g$ in the photo-CIDNP signal enhancement. At $4.7 \mathrm{~T}$ (i.e. $200 \mathrm{MHz}$ ), the $\mathrm{H}_{5}$ signal of trans-HOPI is enhanced 380 -fold in the presence of excited AT12 (triplet at $6.98 \mathrm{ppm}$ ), and $-160-$ fold in the presence of excited fluorescein (Table 1). The tryptophan signal is enhanced by 225 and 90 -fold in the presence of fluorescein and AT12, respectively. The field-dependent normalized photo-CIDNP polarizations have been calculated according to eqn (3), and plotted into Fig. 5. The experimental signal enhancement values were normalized by the relative sensitivity of the $600 \mathrm{MHz}(14.1 \mathrm{~T})$ and $200 \mathrm{MHz}(4.7 \mathrm{~T}) \mathrm{NMR}$ spectrometers to obtain the normalized polarizations, and were fitted to the calculated curves. The photo-CIDNP polarizations monitored with AT12 dye (Fig. 5A) had to be calculated for the different possible $g$-values estimated from the photo-CIDNP anomalous line sign alteration of HOPI, and the $g$-values of tyrosine and tryptophan. ${ }^{13}$ The fitting of the experimental polarization to the calculated curves provided satisfactory results for all the possible $g$-values of AT12 except 2.0036, thus refining the range of $g$-values for HOPI from 2.0037 to 2.0040. Moreover, the optimal field for HOPI is strongly shifted to high field as compared to that of tryptophan, which is explained by the cooperativity of the smaller $\Delta g$ and higher HFCCs as compared to that of tryptophan. The slightly higher field-dependence of the AT12-monitored photo-CIDNP of HOPI as compared to that of tryptophan can be explained by this shift. The fluoresceinmonitored photo-CIDNP field-dependence polarization of HOPI is shifted towards higher field values, due to the particularly low difference in $g$-values (Fig. 5B). Therefore, the optimal field for the HOPI-fluorescein photo-CIDNP polarization is 9-10 T, which

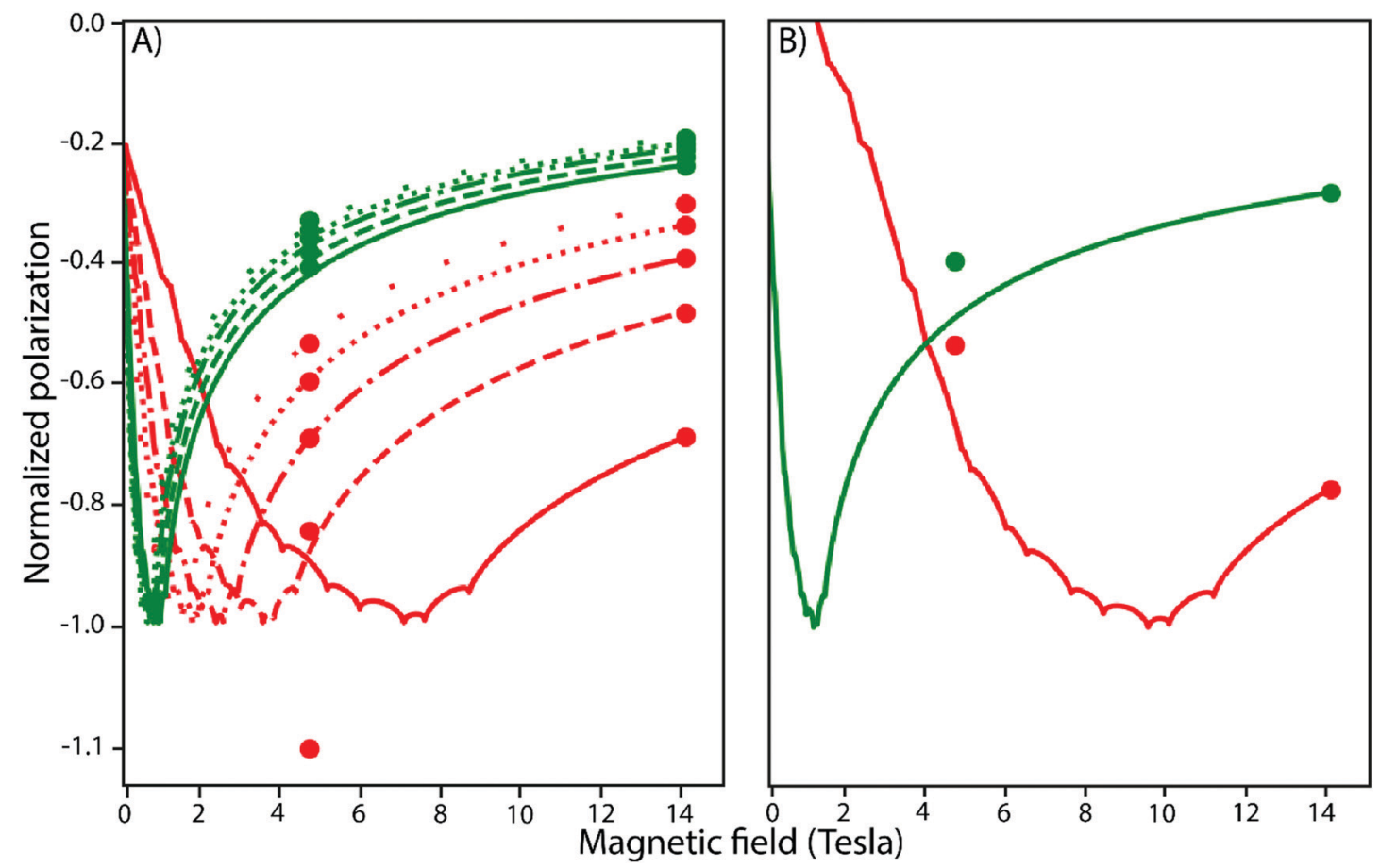

Fig. 5 Field dependent photo-CIDNP polarization calculated from the HFCC of HOPI (red) and tryptophan (green) in the presence of AT12 (A), or fluorescein (B). The $g$-value of AT12 is unknown and the curves were simulated for different potential values: 2.0036 (solid), 2.0037 (dashed), 2.0038 (dotdashed), 2.0039 (dotted), and 2.0040 (loosely dotted). 
would correspond to a $400 \mathrm{MHz}$ spectrometer. This surprising finding is corroborated by the relatively lower polarization of HOPI at $200 \mathrm{MHz}$ as compared to that at $600 \mathrm{MHz}$. In comparison, the tryptophan-fluorescein photo-CIDNP couple has a higher $\Delta g$ and presents a slight increase of the polarization at $200 \mathrm{MHz}$ as compared to that at $600 \mathrm{MHz}$. All these findings together show a stronger field-dependence of the AT12-based enhancement compared to that of fluorescein. This dye-specific fielddependence is in large part explained by the $\Delta g$, the difference of the $g$-factor of the dye to the $g$-factor of the radicals. In principle, at lower fields, the HFCCs have a greater influence and the polarization resulting from each spin sorting step is higher and reaches a maximum when the condition $\mu_{\mathrm{B}} \Delta g B_{0}=$ HFCC/2 is fulfilled. However, as it appears with the HOPI fluorescein couple, this greater influence of the HFCC can be observed at higher field if the $\Delta g$ is small enough. Fig. S11 (ESI $\dagger$ ) shows the ranking of the molecules according to the $g$-factor of their radicals. Fluorescein has a smaller $\Delta g$ with tryptophan than that of AT12 as demonstrated elsewhere. ${ }^{12}$ Furthermore, based on our calculation, the $\Delta g$ of the fluorescein-HOPI pair is expected to be very low and on the order of 0.0001 and thus its effect on the polarization is not very magnetic field-sensitive, while the $\Delta g$ of the AT12-HOPI pair is several times larger yielding a stronger magnetic field-dependence of the polarization. Whether the higher quantum yield of AT12 compared to that of fluorescein is also an important factor in the magnetic field-dependence of polarization is open for discussion. ${ }^{12,30}$ Furthermore, the calculated $g$-factors for the HOPI radicals yielded values in the vicinity of 2.0035 (see the ESI $\dagger$ ). Considering the sign of the anomalous lines, the $g$-factor of the HOPI radicals have been estimated above fluorescein's $g$-factor (2.0034) and below AT12's (between 2.0034 and 2.0041). However, the sign of the polarization when the experiments are conducted with $3,3^{\prime}, 4,4^{\prime}$ tetracarboxy-benzophenone (TCBP) dye with a $g$-factor $=2.0035$ (Fig. S7 and S8, ESI $\dagger$ ) enables one to place the $g$-factor value of HOPIs in between 2.0034 and 2.0035. Furthermore, the polarization of HOPI at $200 \mathrm{MHz}$ in the presence of TCBP is 3-fold lower as compared to that of tryptophan, which is expected since the $\Delta g$ of HOPI with TCBP is estimated to be smaller than the $\Delta g$ of HOPI with fluorescein, and shifts the optimal magnetic field to higher values.

Finally, since the $\Delta g$ between HOPI and fluorescein is lower than the $\Delta g$ between HOPI and AT12, one would expect the HOPI fluorescein couple to yield higher signal enhancements, while considering only the magnetic parameters. However, it is the AT12, which provides the best performance as a photoCIDNP sensitizer for HOPI, suggesting that other parameters than magnetics are to be considered to address the polarization quantitation.

\section{Hyperfine couplings}

Despite the fact that triplet-singlet state mixing of the radical pair electron spins are mainly driven by the $\Delta g$ in fields above 1 Tesla (high-field), the spin sorting, and thus the hyperpolarization is the result of the HFCCs. These coupling constants are not field-dependent and are specific for each nucleus within the radical. The HFCC values depend on the Fermi contact interaction between the nucleus and the unpaired electron, and on the spin density. ${ }^{33}$ As a consequence of the differences in the conjugated systems of tryptophan and HOPI, their spin densities are different. Unlike the absorptive signals of the aromatic protons of tryptophan, the aromatic anomalous signals are emissive and absorptive for HOPI. Moreover, the $\mathrm{H}_{2}$ proton, which was aromatic in the tryptophan is now in the $\alpha$-position of the conjugated system and has an intense emissive signal after irradiation in the presence of AT12. To get further insights into the HFCC constants of HOPI with an emphasis on understanding which of the chemical features are important to generate a highly polarizable molecule, HFCCs were computed from DFT simulations run on Gaussian (Table $\mathrm{S} 2, \mathrm{ESI} \dagger)$.

In order to determine experimentally the HFCC and mechanistic insights (see below) time-resolved (tr) photoCIDNP is necessary because in a CW-photo-CIDNP experiment the predominance of F-pair over geminate polarization (slightly) deviates from the total polarization and only the geminate polarization is purely related to the HFCC. ${ }^{34}$ For TR-photo-CIDNP the experimental conditions were changed (see the ESI for details) using TCBP as the dye because the hyperfine couplings of its radicals have been determined from $\mathrm{EPR}^{35}$ and $\mathrm{CIDNP}^{23,36}$ and because its radicals show clear CIDNP patterns that are distinctly different for electron transfer (ET) and proton coupled electron transfer (PCET) reactions. The experiments were performed at $4 \mathrm{mM}$ concentration of HOPI and at a $\mathrm{pH}$ of 7.5 (Fig. S7 and S8, ESI $\dagger$ )

The experimental HFCCs determined by the TR-photoCIDNP anomalous line intensities (Fig. S7 and S8, ESI $\dagger$ ) were then correlated with the calculated ones shown in Fig. 6A and B showing determination coefficients of 0.97 and 0.98 for transHOPI and cis-HOPI, respectively (Fig. 6A and B). It is thereby interesting to note that the $\mathrm{H}_{2}$ HFCC was dramatically increased (i.e. by a factor of 5-6) by the aromaticity loss of the five membered-ring. The high HFCC of beta protons in tyrosine and tryptophan have already been predicted. However, because of the angular dependence of the HFCC, ${ }^{37}$ the rotation around the $\sigma$ molecular bond was averaging out the expected hyperpolarization effect. From this observation, we conclude that in general, aliphatic protons in the $\alpha$ position to an aromatic system with hindered rotational mobility (i.e. that in a cyclic aliphatic ring) yield high HFCC.

\section{Electron transfer}

Since the HFCC of the TCBP dye depend on its protonation state, the electron transfer mode can be addressed by analyzing the TR-photo-CIDNP anomalous line intensities. ${ }^{23}$ In the case of tryptophan or $\mathrm{N}$-acetyl tryptophan, the TCBP anomalous line intensities match with the unprotonated TCBP's HFCCs and thus a simple electron transfer (ET). However, the time-resolved CIDNP spectra for cis/trans-HOPI yield a mix of protonated TCBP radicals as it can be observed from the correlation plot between the anomalous line intensities and the HFCCs of 

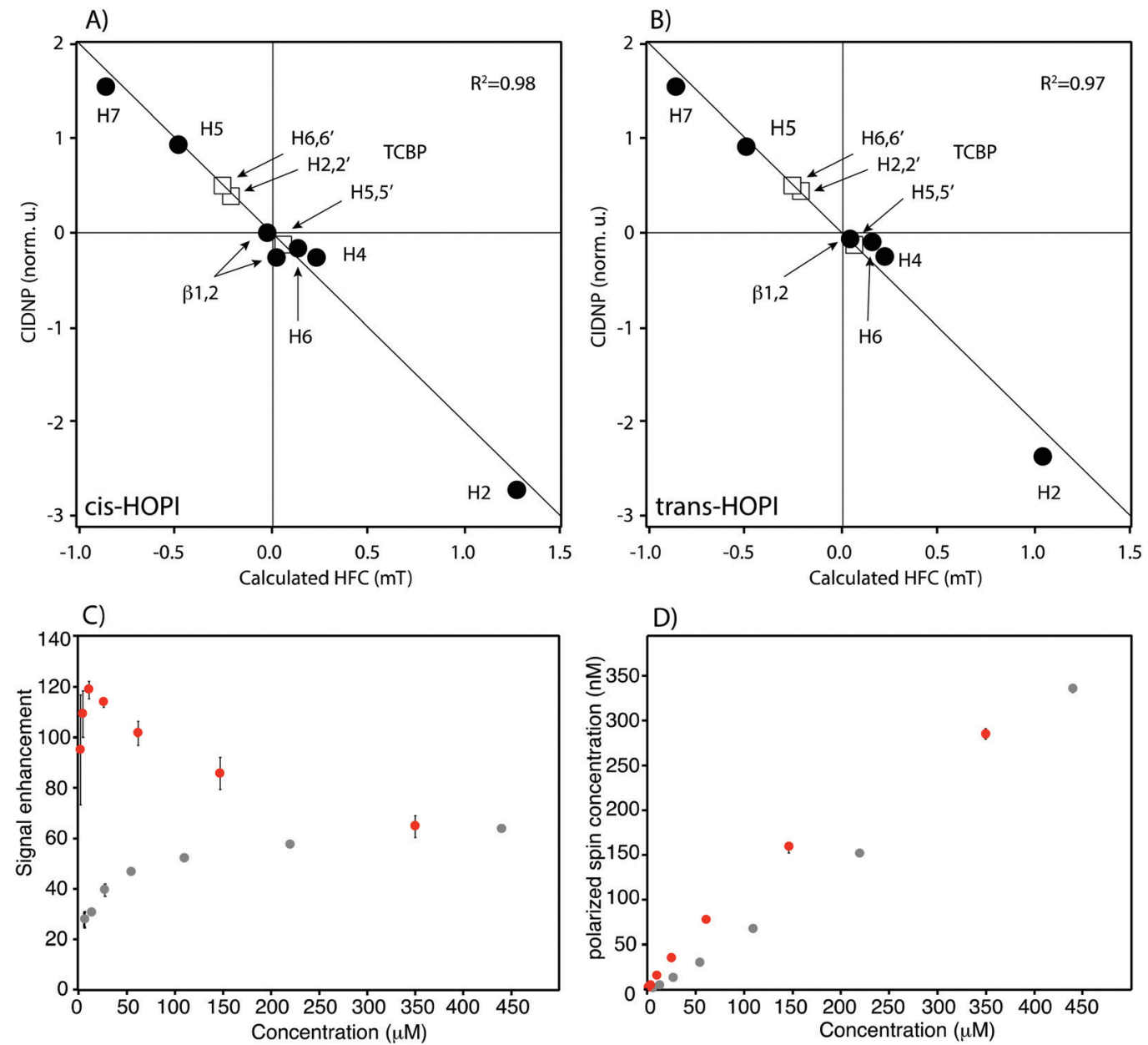

Fig. 6 Correlation between the ${ }^{1} \mathrm{H}$ CIDNP intensities of the geminate products detected in the reversible photoreaction of TCBP and the calculated ${ }^{1} \mathrm{H}$ HFCCs of the cis-HOPI (A) and trans-HOPI (B) radicals (circles) and the combination of TCBP radicals I, III, and IV (squares). Solid line: best fit by the function P1 $=-$ CA1i (fitting to circles), P2j $=-$ CA2j (fitting to squares). Concentration-dependent H5 Photo-CIDNP enhancement for trans- and cis-HOPI. (C) Concentration-dependence of the enhancement of trans-HOPI shown as red circles and cis-HOPI data shown in grey circles. (D) Concentration dependence of the polarized spin concentration. For the calculation it is assumed that the polarization at $14.1 \mathrm{~T}$ was 100 ppm. The data points shown in red are: trans-HOPI $(350,175,88,44,22,11$, and $5.5 \mu \mathrm{M})$ and in grey: cis-HOPI $(440,220,110,55,27.5,13.8, a n d 6.9 \mu \mathrm{M})$.

TCBP $/ \mathrm{H}^{+}$mix (Fig. 6A, B and Fig. S9, S10, Table S2, ESI $\dagger$ ). This last observation suggests a PCET reaction mechanism for the HOPI radicals. The degenerate electron exchange takes place when the radical and the diamagnetic molecule differ only by one electron. It is worth noting that PCET is also the formation mechanism of tyrosyl radicals, ${ }^{23}$ which also shows improved signal enhancement when photo-CIDNP experiments are performed in the presence of AT12. ${ }^{13}$

\section{Diastereomer-dependent reaction kinetics}

Under the given experimental conditions, the photo-CIDNP enhancement of the $\mathrm{H}_{5}$ signal is similar for trans-HOPI and cis-HOPI (73 versus 68, Table 1). However, the signal enhancement of the $\mathrm{H}_{2}$ proton is different for the two diastereoisomers, and each exhibits two different chemical shifts that correspond to two slowly interconverting conformers. The larger enhancement factor for trans-HOPI $\mathrm{H}_{2}$ is -82-fold for its upfield conformer and for cis-HOPI $\mathrm{H}_{2}$ the larger enhancement is -47-fold for its downfield conformer. Moreover, the HOPI concentration-dependence of the signal enhancement displays completely different behaviours for the two diastereoisomers. At the lower concentration of $20 \mu \mathrm{M}$, trans-HOPI shows an enhancement of 120 for the $\mathrm{H}_{5}$ resonance, whereas for cis-HOPI it is only ca. 40 (Fig. 6C). Towards an understanding of this difference it is noted that the radical pair formation obeys bimolecular steady state kinetics, which is outlined in the ESI $\uparrow$ "Reaction kinetics". It is demonstrated that the slope of the concentration-dependent photo-CIDNP spin polarization shown in Fig. 4D provides the time-independent rate constant ratio $k_{1} / k_{-1}$ under the assumption that both the HOPIs are in surplus, where $k_{1}$ is the radical pair formation rate constant and $k_{-1}$ the overall radical pair recombination rate constant. Based on the fits of the experimental data from Fig. $4 \mathrm{D}$, this ratio is higher for trans-HOPI $\left(54 \mathbf{M}^{-1}\right)$ than cis-HOPI $\left(39 \mathbf{M}^{-1}\right)$.

Similar mechanistic insights are obtained from competition experiments with photo-CIDNP of tryptophan with either cisHOPI or trans-HOPI with the latter showing a stronger interference on the photo-CIDNP of tryptophan (Fig. S6, ESI $\dagger$ ). In principle, the conformational dependence of the $g$-factors and 
the HFCCs could modulate the recombination rate $k_{-1}$. However, neither the HFCC nor the $g$-factor calculations showed a significant difference between the two diastereoisomers (Tables S2 and S3, ESI $\dagger$ ) suggesting that the reason for different polarization properties is solely resulting from the reaction yield of the radical pair formation. This suggests other modulation parameters such as the conformational flexibility, redox potential, ${ }^{38}$ hydrodynamic radius or the dye-HOPI interaction potential to be the key for the different polarizations observed. We propose here a straightforward explanation referring to the Marcus theory for electron transfer, or in this case proton coupled electron transfer. ${ }^{39,40}$ Shortly, according to the FranckCondon principle, the electron transfer timescale being orders of magnitude faster than the nuclei motions, the radicals are generated in the wrong solvation environment, and in the wrong vibrational state. Marcus revised the well-known Franck-Condon principle to satisfy energy conservation. Hence, the vibrational states of the diamagnetic reactant must match the vibrational states of its radical product to enable the electron transfer. The quantum mechanics simulations predict that the cis-HOPI has an internal $\mathrm{H}$-bond between the hydroxy and the carboxy groups, while the trans-HOPI does not. However, $T_{1}$ relaxation experiments revealed no decrease of the relaxation time $T_{1}$ for $\mathrm{cis}$-HOPI as compared to that of trans-HOPI with $T_{1}$ values of $3.88 \mathrm{~s}$ and $3.26 \mathrm{~s}$, respectively. This last experiment invalidates the $\mathrm{H}$-bond hypothesis. Nevertheless, the HPLC retention times suggest that the trans diastereoisomer may be less hydrophobic than the cis. This difference in hydrophobicity is very likely related with a different solvation profile and may be the result of the different conformations. This particular conformation of the cis-HOPI interacts differently with its environment and is responsible for a higher energy penalty, while jumping to the higher vibrational states as compared to the trans-HOPI. The result of this is a higher free energy barrier for the generation of the radical in the case of the cis-HOPI and then a slower reaction rate according to eqn (3):

$$
k_{\mathrm{et}}=A \mathrm{e}^{\frac{-\Delta G^{*}}{k_{\mathrm{b}} T}}
$$

The differences in the reactivities of the two diastereoisomers and their impact on the hyperpolarization efficacy suggest that diastereoisomeric compounds have to be tested separately regarding their photo-CIDNP performance. Because of the higher energy and much shorter life time of the TCBP triplet state, this high performance was not observed during the TR-photo-CIDNP experiments at $\mathrm{mM}$ concentration in the presence of TCBP. Under these conditions the two HOPI compounds showed lower rate constants for quenching of the dye and lower enhancements as compared to those of tryptophan and $\mathrm{N}$-acetyltryptophan (Fig. S7, $\mathrm{ESI} \dagger$ ). Furthermore, no significant difference in the signal intensities could be observed. This emphasizes the fact that for $\mathrm{CW}$-photo-CIDNP experiments at $\mu \mathrm{M}$ concentration the polarization performance depends on the dye-molecule couple.

\section{On the AT12-HOPI pair - an outlook}

The present study starting from a serendipitous observation identified a powerful CIDNP dye-ligand pair - AT12 and HOPI - yielding at relatively low concentrations signal enhancement in the order of two to three orders of magnitude, explained by the symbiotic contributions of several factors such as hyperfine couplings, $g$-factor difference between dye and ligand and reaction kinetics. This pair opens on one hand an avenue towards high throughput screening or NMR experiments at very low concentrations of ligand protein interaction studies relevant in biological sciences and drug research, if the HOPI can be covalently linked as a CIDNP-NMR probe to the molecules of interest as usually required in fluorescencebased experiments/assays. The HOPI is thereby a rather small molecule when compared to the usual fluorescent dyes. Moreover, HOPI photo-CIDNP can be monitored with AT12, which is excited at $532 \mathrm{~nm}$, an important redshift when compared to fluorescein $(450 \mathrm{~nm})$, FMN (450 nm), bipyridyl (308 nm), or TCBP (308 nm). On the other hand, the HOPI studies indicate that many small molecules of chemical and biological interest are potentially CIDNP-active as they comprise more often than rare tryptophan-like or HOPI-like entities showing that there is a significant chemical space of CIDNP-active molecules to be investigated and potentially used.

\section{Conclusions}

Understanding the chemical features that make photo-CIDNP highly efficient is of great value in designing highly polarizable radical pairs. The previously reported maximum ${ }^{1} \mathrm{H}$ signal enhancement was for tyrosine, ${ }^{13}$ which could be enhanced up to 60 -fold with AT12, while trans-HOPI could be polarized up to 120 -fold at a magnetic field of $14.1 \mathrm{~T}$. At a magnetic field of 4.7 $\mathrm{T}$ the ${ }^{1} \mathrm{H}$ signal enhancement observed is up to 380 -fold and the ${ }^{13} \mathrm{C}$ enhancement at $14.1 \mathrm{~T}$ is estimated to be on the order of 1000-fold. The optimal field for the fluorescein-HOPI pair was estimated to be $400 \mathrm{MHz}$ and improved photo-CIDNP performances are expected at this field. In addition, the optimal field for the HOPI-TCBP is potentially higher than $400 \mathrm{MHz}$. The optimization of photo-CIDNP pairs for high field could yield to unprecedented signal to noise performance. The reasons for such enhancements are the larger HFCCs, reduced $\Delta g$ and improved yields of the reactive species. It was already observed that aliphatic protons in the alpha position relative to an aromatic cycle presented the highest HFCC. Unfortunately, the dihedral-dependence of the HFCC combined with the rotation of the aliphatic bonds was averaging these HFCCs to lower values. ${ }^{33,41-43}$ The constraining of the aliphatic proton $\mathrm{H}_{2}$ in a 5-membered ring impedes the rotation and provides a high HFCC that is responsible for a high photo-CIDNP signal enhancement. Moreover, smaller conjugated systems generate higher spin density and higher HFCCs, as well as modify the SOMO shape and the resulting HFCCs and $g$-factors. Furthermore, the difference in signal enhancement for the two diastereoisomers underlines the importance of the reactivity of the molecule in relation to the dye. It is an elegant illustration of the Marcus theory and suggests that diastereoisomers should be investigated separately. Furthermore, given the intrinsic 
complexity of the CIDNP phenomenon, the selection of a dyemolecule couple for CW-photo-CIDNP application cannot be simply derived from the quenching rate and magnetic parameters obtained with TR-photo-CIDNP. The latter is necessary to obtain the information about the electron transfer reaction and the HFCC. It gives also a clear idea of the quenching rates in play. Therefore, it is concluded that the selection of an optimal dyepolarizable aromatic molecule pair may yield unprecedented signal enhancement on the order of several orders of magnitude.

\section{Author contributions}

F. T. performed most of the experiments and wrote the manuscript. A. S. made the first observation of the HOPI lines. J. G. helped to identify the oxidation product of tryptophan. A. R. helped with the experiments and wrote the manuscript. O. M. performed and analysed the TR-photo-CIDNP experiments. A. Y. helped with analysing TR-photo-CIDNP results. R. R. helped with the experiments and wrote the manuscript.

\section{Conflicts of interest}

There are no conflicts to declare.

\section{Acknowledgements}

We would like to thank Daniel Zindel for the synthesis of the $3 \alpha$-hydroxypyrroloindole diastereoisomer. Y. A. and O. M. acknowledge support by Russian Foundation for Basic Research (projects 20-03-00234 and 19-29-10028).

\section{References}

1 A. Abragam, The principles of nuclear magnetism, Clarendon Press, Oxford, 1961.

2 J. H. Ardenkjaer-Larsen, K. Golman, A. Gram, M. H. Lerche, R. Servin, M. Thaning and J. Wolber, Discovery Med., 2003, 3, 37-39.

3 C. R. Bowers and D. P. Weitekamp, Phys. Rev. Lett., 1986, 57, 2645-2648.

4 R. W. Adams, J. A. Aguilar, K. D. Atkinson, M. J. Cowley, P. I. Elliott, S. B. Duckett, G. G. Green, I. G. Khazal, J. LopezSerrano and D. C. Williamson, Science, 2009, 323, 1708-1711.

5 R. W. Adams, S. B. Duckett, R. A. Green, D. C. Williamson and G. G. Green, J. Chem. Phys., 2009, 131, 194505.

6 J. Bargon, H. Fischer and U. Johnsen, Z. Naturforsch. A., 1967, A 22, 1551-1555.

7 H. R. Ward and R. G. Lawler, J. Am. Chem. Soc., 1967, 89, 5518-5519.

8 G. L. Closs, J. Am. Chem. Soc., 1969, 91, 4552-4554.

9 R. Kaptein and J. L. Oosterhoff, Chem. Phys. Lett., 1969, 4, 195-197.

10 F. J. Adrian, J. Chem. Phys., 1971, 54, 3912-3917.

11 R. M. Noyes, J. Chem. Phys., 1954, 22, 1349-1359.

12 Y. Okuno and S. Cavagnero, J. Phys. Chem. B, 2016, 120, 715-723.
13 A. Sobol, F. Torres, A. Aicher, A. Renn and R. Riek, J. Chem. Phys., 2019, 151, 234201.

14 Y. Okuno and S. Cavagnero, J. Magn. Reson., 2018, 286, 172-187.

15 Y. Okuno and S. Cavagnero, Emagres, 2017, 6, 283-313.

16 O. B. Morozova and K. L. Ivanov, ChemPhysChem, 2019, 20, 197-215.

17 M. Cocivera, J. Am. Chem. Soc., 1968, 90, 3261-3263.

18 G. L. Closs, in Advances in Magnetic and Optical Resonance, ed. J. S. Waugh, Academic Press, San Diego, 1974, vol. 7, pp. 157-229.

19 J. K. Vollenweider, H. Fischer, J. Hennig and R. Leuschner, Chem. Phys., 1985, 97, 217-234.

20 J. K. Vollenweider and H. Fischer, Chem. Phys., 1986, 108, 365-372.

21 J. K. Vollenweider and H. Fischer, Chem. Phys., 1988, 124, 333-345.

22 A. S. Kiryutin, K. L. Ivanov, O. B. Morozova, A. V. Yurkovskaya, H. M. Vieth, Y. A. Pirogov and R. Z. Sagdeev, Dokl. Phys. Chem., 2009, 428, 183-188.

23 O. B. Morozova, K. L. Ivanov, A. S. Kiryutin, R. Z. Sagdeev, T. Kochling, H. M. Vieth and A. V. Yurkovskaya, Phys. Chem. Chem. Phys., 2011, 13, 6619-6627.

24 J. H. Lee and S. Cavagnero, J. Phys. Chem. B, 2013, 117, 6069-6081.

25 G. E. Ronsein, M. C. de Oliveira, M. H. de Medeiros and P. Di Mascio, J. Am. Soc. Mass Spectrom., 2009, 20, 188-197.

26 M. Gracanin, C. L. Hawkins, D. I. Pattison and M. J. Davies, Free Radical Biol. Med., 2009, 47, 92-102.

27 W. E. Savige, Aust. J. Chem., 1975, 28, 2275-2287.

28 G. E. Ronsein, M. C. B. Oliveira, S. Miyamoto, M. H. G. Medeiros and P. Di Mascio, Chem. Res. Toxicol., 2008, 21, 1271-1283.

29 M. Nakagawa, S. Kato, S. Kataoka, S. Kodato, H. Watanabe, H. Okajima, T. Hino and B. Witkop, Chem. Pharm. Bull., 1981, 29, 1013-1026.

30 A. Chmyrov, J. Arden-Jacob, A. Zilles, K. H. Drexhage and J. Widengren, Photochem. Photobiol. Sci., 2008, 7, 1378-1385.

31 J. H. Lee, A. Sekhar and S. Cavagnero, J. Am. Chem. Soc., 2011, 133, 8062-8065.

32 R. Kaptein, J. Chem. Soc., Chem. Commun., 1971, 732-733.

33 R. Improta and V. Barone, Chem. Rev., 2004, 104, 1231-1253.

34 A. S. Kiryutin, O. B. Morozova, L. T. Kuhn, A. V. Yurkovskaya and P. J. Hore, J. Phys. Chem. B, 2007, 111, 11221-11227.

35 O. Säuberlich, J. Brede and D. Beckert, J. Phys. Chem., 1996, 100, 18101-18107.

36 O. B. Morozova, M. S. Panov, N. N. Fishman and A. V. Yurkovskaya, Phys. Chem. Chem. Phys., 2018, 20, 21127-21135.

37 A. Torolabbe and J. Maruani, J. Magn. Reson., 1985, 61, 254-261.

38 D. Rehm and A. Weller, Isr. J. Chem., 1970, 8, 259-271.

39 R. A. Marcus, J. Chem. Phys., 1956, 24, 979-989.

40 R. A. Marcus, J. Chem. Phys., 1956, 24, 966-978.

41 I. Kuprov, T. D. Craggs, S. E. Jackson and P. J. Hore, J. Am. Chem. Soc., 2007, 129, 9004-9013.

42 F. Khan, I. Kuprov, T. D. Craggs, P. J. Hore and S. E. Jackson, J. Am. Chem. Soc., 2006, 128, 10729-10737.

43 I. Kuprov and P. J. Hore, J. Magn. Reson., 2004, 168, 1-7. 